\title{
Study on Ballistic Missile Infrared Hyperspectral Endmember Extraction Algorithm Based on Non- negative Matrix Factorization—Feasibility Analysis
}

\author{
Shihua Liu, Jiangtao Xiang and Wei Qi \\ Dept. 3, AFEWA, Wuhan 430019, China
}

\begin{abstract}
According to the ballistic missile infrared hyperspectral endmember extraction problem, a hyperspectral endmember extraction algorithm based on the non-negative matrix factorization is studied. This algorithm is solved based on the alternating least square. The signal separation and extraction ability of this algorithm is good. In the simulation, the algorithm feasibility and the effect that the hybrid spectral number to the algorithm performance are mainly researched. The simulation show that the algorithm has good performance in the endmember extraction.
\end{abstract}

Keywords-endmember extraction; non-negative matrix factorizatio; ballistic missile; infrared hyperspectral

\section{INTRODUCTION}

Let each spectral detected by the spectrometer as a pixel, the target area correspond to each pixel may conclude different materials, that is, each pixel is a mixed pixel, its corresponding spectral is a mixture of different material spectral. In order to analyse the material kinds of the target area and the proportion of each material in the area, the endmember extraction is needed to the spectral, the different material spectral and the corresponding proportion of each mixed pixel is needed to computed. The material spectral is named as endmember, the proportion of each endmember is named as abundance. The endmember extraction is an important content in the hyperspectral remote sensing.

In order to solve the endmember extraction problem, many feasible methods have been presented. Such as, the pure pixel index algorithm(PPI)[1], the N-FINDR algorithm[2], the vertex component analysis algorithm(VCA)[3], the simplex growing algorithm(SGA)[4], the Null space spectral projection algorithm[5]. In these methods, the various material pixel in the image is assumed based on the linear spectral mixture mdel. In recent years, some endmember extraction algorithms are presented based on the spatial information and spectral information. For example, the automated morphological endmember extraction algorithm(AMEE), the spatial-spectral endmember extraction algorithm(SSEE), the spatial purity based endmember extraction algorithm(SPEE).

In despite of the number of endmember extraction algorithm is not less, but most of the methods are situated to the image-level endmember extraction and not to the spectral- level endmember extraction. In this paper, the study object is the infrared spectral, the above methods are not suitably. Aim at the study object in this paper, the non-negative matrix factorization algorithm(NMF) is researched.

\section{INFRARED ULTRASPECTRAL ENDMEMBER EXTRACTION BASED ON THE NON-NEGATIVE MATRIX FACTORIZATION}

The problem of the non-negative matrix factorization can be represent as follows[4]: Given a non-negative matrix $\mathbf{A} \in \mathbf{R}^{n \times m}$ and a positive integer $k \leq \min \{n, m\}$, two nonnegative matrixes $\mathbf{W} \in \mathbf{R}^{n \times k}$ and $\mathbf{H} \in \mathbf{R}^{k \times m}$ are needed to satisfied with

\section{$\mathbf{A} \approx \mathbf{W H}$}

In order to scale the similarity between $\mathbf{W H}$ and $\mathbf{A}$, an objective function is need to present. The common objective function is the Euclidean distance, thai is,

$$
f(\mathbf{W}, \mathbf{H})=\frac{1}{2}\|\mathbf{A}-\mathbf{W H}\|_{\mathrm{F}}^{2}
$$

When $\mathbf{A}=\mathbf{W H}$, the objective function is smallest. So the non-negative matrix factorization problem is changed to find two non-negative matrixes to make $f(\mathbf{W}, \mathbf{H})$ smallest. The two non-negative matrixes are named as the $k$-order nonnegative matrix factorization, where $k$ is a known number and $k=\min \{n, m\}$.

The numerical solution of the NMF problem can be classified as follows: the gradient descent algorithm(GDA), the multiplicative update algorithm(MUA) and the alternating least square algorithm(ALS). In this paper, the ALS is mainly researched.

The principle of ALS is: the object function is non-convex to $\mathbf{W}$ and $\mathbf{H}$, but the single $\mathbf{W}$ and $\mathbf{H}$ are all convex 
function. In ALS, fix $\mathbf{W}$ and $\mathbf{H}$ in turn and compute another number by the alternating least square algorithm and repeat the above process.

Aim at the local minimum value problem of NMF, there are many improved algorithms are presented. In these algorithms, most of them to avoid the algorithms traped in the local minimum value by additional constraint conditions. Hoyer consider the sparsity of the coefficient matrix and present the non-negative matrix factorization algorithm with sparsity constraint. This method join the constraint condition in the object function, which change the object function as

$$
f(\mathbf{W}, \mathbf{H})=\frac{1}{2}\|\mathbf{A}-\mathbf{W H}\|_{\mathrm{F}}^{2}+\alpha \sum_{i, j} h_{i j}
$$

In this constraint condition, the sparsity is expressed as the sum of all elements in matrix $\mathbf{H}$. In the Iteration process, the sparsity is reached to smallest to make the factorization output is close to the true value.

Otherwise, Pauca and Piper introduce the constraint conditions of the smoothness of $\mathbf{W}$ and the sparsity of $\mathbf{H}$ into ALS, which change the object function as

$$
f(\mathbf{W}, \mathbf{H})=\frac{1}{2}\|\mathbf{A}-\mathbf{W H}\|_{\mathrm{F}}^{2}+\frac{\alpha}{2}\|\mathbf{W}\|_{\mathrm{F}}^{2}+\frac{\beta}{2}\|\mathbf{H}\|_{\mathrm{F}}^{2}
$$

From the above we can conclude that what constraint condition are need to be used should decided by the research problem. In the spectral endmember extraction problem, it is not only have the characteristic of non- negative, but also satisfied the condition that the sum of the total coefficients must equal to 1 . Therefore, a constraint condition introduced into the object function

$$
f(\mathbf{W}, \mathbf{H})=\frac{1}{2}\|\mathbf{A}-\mathbf{W H}\|_{\mathrm{F}}^{2}+\frac{\alpha}{2}\left\|\mathbf{H}^{\mathrm{T}} \mathbf{e}_{1}-\mathbf{e}_{2}\right\|_{\mathrm{F}}^{2}
$$

Where $\mathbf{e}_{1}=\mathbf{1}^{k \times 1}, \mathbf{e}_{2}=\mathbf{1}^{m \times 1}$ and $\alpha$ is the chastening factor. $\mathbf{H}^{\mathrm{T}} \mathbf{e}_{1}-\mathbf{e}_{2}=0$ is reach to the smallest when every column sum is 1 . Hence, the gradients of $\mathbf{W}$ and $\mathbf{H}$ of $f(\mathbf{W}, \mathbf{H})$ is recomputed

$$
\begin{aligned}
& \frac{\partial f(\mathbf{W}, \mathbf{H})}{\partial \mathbf{W}}=-(\mathbf{A}-\mathbf{W H}) \cdot \mathbf{H}^{\mathrm{T}} \\
& \frac{\partial f(\mathbf{W}, \mathbf{H})}{\partial \mathbf{H}}=-\mathbf{W}^{\mathrm{T}} \cdot(\mathbf{A}-\mathbf{W H})+\alpha \cdot \mathbf{e}_{1}\left(\mathbf{e}_{1}^{\mathrm{T}} \mathbf{H}-\mathbf{e}_{2}^{\mathrm{T}}\right)
\end{aligned}
$$

Apply the above equations into the ALS and the improved ALS is achieved.

\section{SIMULATION AND ANALYSIS}

The purpose of this paper is to simulate the endmember extraction of the ballistic missile infrared spectral in the deep space. The ballistic missile is into the field of view when the position of the ballistic missile is captured. Because we study the midcourse characteristic of the ballistic missile, so the object spectral characteristics are mainly conclude the skin spectral of the ballistic missile and the deep space spectral. Meanwhile, the spectrals in different conditions of the ballistic missile are the spectrals that captured in different time points. In this paper, the condition of random hybrid abundances is mainly researched. The research of other conditions is introduced in other papers.

The spectrals in the experiment are simulated by the computer, which the wavenumber range is from $671 \mathrm{~cm}-1$ to $5000 \mathrm{~cm}-1$, the points number is $n=4324$. The original skin spectral and the hybrid spectral of the target are shown as Figure I and Figure II. Because the hybrid spectral is composed of the skin spectral and the background spectral, so $k=2$ and $\alpha=2$. The maximal iterative time is 1000 .

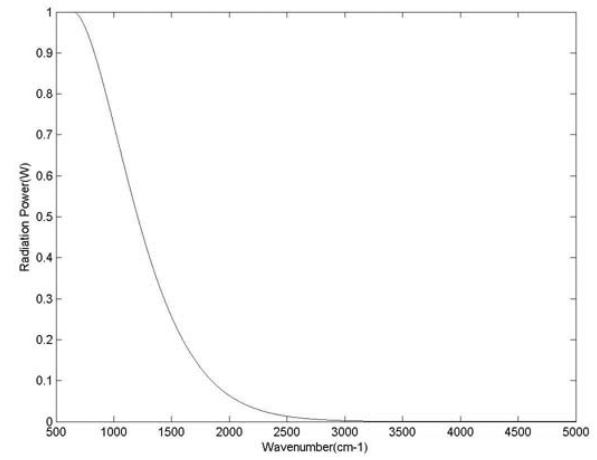

FIGURE I. THE ORIGINAL SKIN SPECTRAL

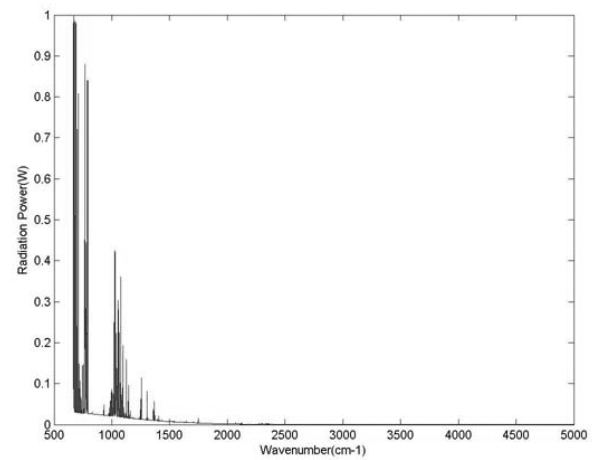

FIGURE II. THE HYBRID SPECTRAL

Constringency conditions:

1. The difference of mean square errors of $\mathbf{A}-\mathbf{W H}$ in adjacent iteration is less than $10^{-9}$; 
2. The differences of mean square errors of $\mathbf{W}$ or $\mathbf{H}$ in adjacent iteration are less than $10^{-9}$;

\section{The iterative time is beyond the maximum.}

The precondition of this hybrid method is that the abundances of the skin spectral and the background spectral are not correlative in different time points, that is, the aboundance of each endmember is similar to the white noise. When the hrbrid spectral number is $m=100$, the endmember extraction output of the skin spectral and the different between the extracted spectral and the original spectral are shown as Figure III and Figure IV.

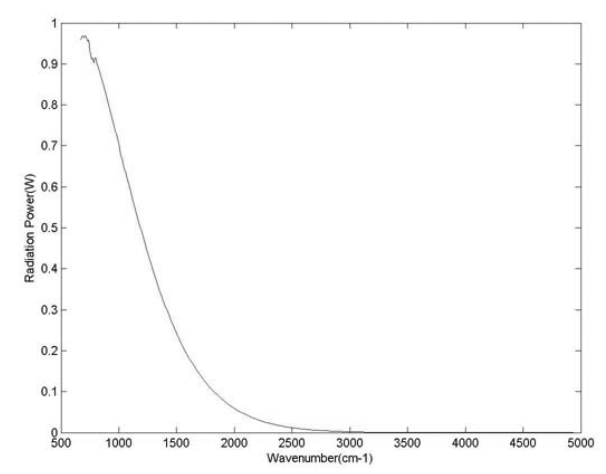

FIGURE III. THE EXTRACTED SKIN SPECTRAL

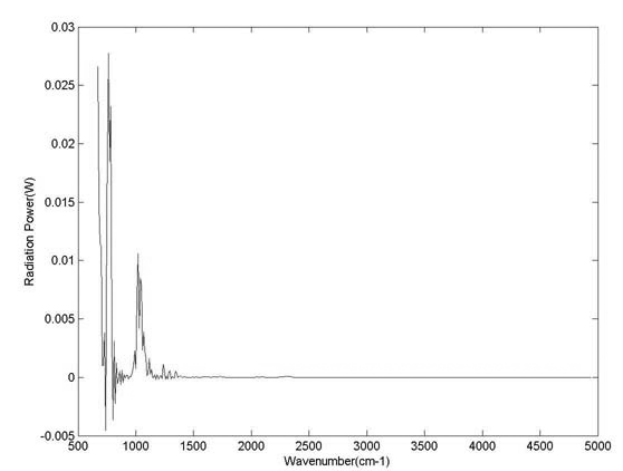

FIGURE IV. THE DIFFERENCE SPECTRAL

From Figure III and Figure IV we can conclude that the extracted skin spectral after endmember extraction is approximated the original skin spectral. In general, the difference of them is small. the distinct error is only in the wavenumber range of $700 \mathrm{~cm}^{-1} \sim 800 \mathrm{~cm}^{-1}$ and $1000 \mathrm{~cm}^{-}$

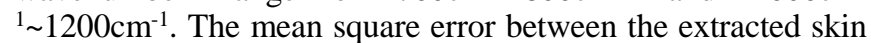
spectral and the original skin spectral is $2.8302 \times 10^{-3}$.

The hybrid spectrals provided more information quantity and the endmember extracted effect is better when more hybrid spectrals are obtained. Oppositely, the endmember extracted effect is worse when the hybrid spectrals are less. Subsequently, the endmember extracted effect is farther researched in the condition that the hybrid spectrals are less.

Considering that the number of the hybrid spectrals is $m=10$, then the endmember extraction output of the skin spectral and the different between the extracted spectral and the original spectral are shown as Figure V and Figure VII. The mean square error between the extracted skin spectral and the original skin spectral is $9.2091 \times 10^{-3}$.

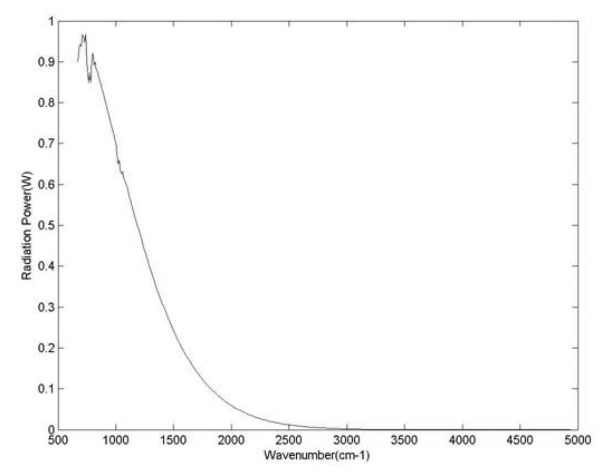

FIGURE V. THE EXTRACTED SKIN SPECTRAL

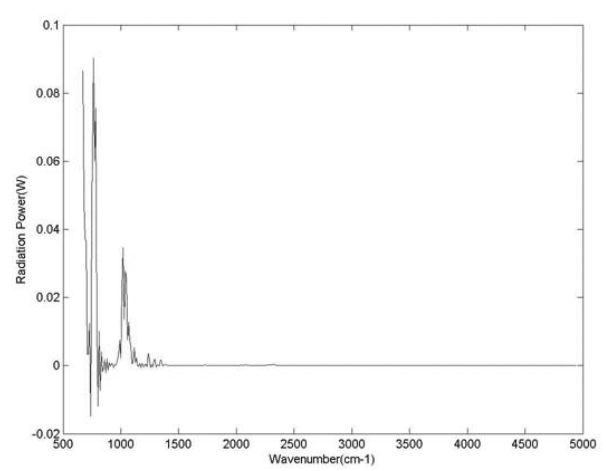

FIGURE VI. THE DIFFERENCE SPECTRAL

In the above figures, the information quantity is reduced while the hybrid spectral is decreased. Meanwhile, similar to the above analysis, the algorithm performance is getting worse. The difference between the extracted skin spectral and the original skin spectral is increased, but the distinct error band is same as before, that is, in the wavenumber range of $700 \mathrm{~cm}^{-}$ ${ }^{1} \sim 800 \mathrm{~cm}^{-1}$ and $1000 \mathrm{~cm}^{-1} \sim 1200 \mathrm{~cm}^{-1}$. In general, the spectral in Figure III is similar in Figure V, both of them are all smooth. If this characteristic is prominent in the following research, this endmember extraction output is acceptable.

\section{CONCLUSIONS}

In this paper, the ballistic missile hyperspectral endmember extraction algorithm based on the NMF is studied. The endmember extraction algorithm based on NMF is not need endmember library and adapt to more situation. However, this algorithm need to consider the ballistic missile hybrid spectrals in different statements. The algorithm feasibility and the effect that the hybrid spectral number to the algorithm performance are mainly researched. The simulation show that this algorithm is adapted to the ballistic missile hyperspectral endmember extraction and the algorithm performance is better in the condition of random hybrid abundances set mode. 


\section{REFERENCES}

[1] J. Guo, Y. S. Li, F. Yi, "FPGA implement for computing pixel purity index of hyperspectral images", Journal of South China University Of Technology(Natural Science Edition), vol. 42(1), pp. 84-89, 2014.

[2] J Qian, K. Z. Deng, D. Liu, "Mineral identification based on the optimized N-FINDR algorithm of hyperspectral remote sensing images", Metal Mine, vol. (8), pp. 84-88, 2012.

[3] L. J. Fang, Y. J. Nian, S. T. Lei, "Endmembers extraction for hyperspectral images based on vertex component analysis", Ship Electronic Engineering, vol. 34(8), pp. 154-158, 2014.

[4] Y. Ma, "Study on targets detection and identification system of infrared hyperspectral imaging", Wuhan, Huazhong University of Science and Technology, 2012.

[5] W. F. Luo, L. Zhong, B. Zhang, Null space spectral projection algorithm for hyperspectral image endmember extraction, J. Infrared Millim. Waves, vol. 29(4), pp. 307-311, 2010. 